\section{(2) OPEN ACCESS}

\title{
Homozygous variants in SYCP2L cause premature ovarian insufficiency
}

\author{
Wen-Bin He, ${ }^{1,2,3}$ Chen Tan, ${ }^{1}$ Ya-Xin Zhang, ${ }^{1}$ Lan-Lan Meng, ${ }^{2,3}$ Fei Gong, ${ }^{1,2,3}$ \\ Guang-Xiu Lu, ${ }^{1,2,3}$ Ge Lin, 1,2,3 Juan Du, ${ }^{1,2,3}$ Yue-Qiu Tan (D) 1,2,3
}

- Additional material is published online only. To view, please visit the journal online (http://dx.doi.org/10.1136/ jmedgenet-2019-106789).

${ }^{1}$ Institute of Reproductive and Stem Cell Engineering, School of Basic Medical Science, Central South University, Changsha 410078, Hunan, China ${ }^{2}$ Reproductive and Genetic Hospital CITIC Xiangya, Changsha 410078, Hunan, China

${ }^{3}$ Clinical Research Center For Reproduction and Genetics In Hunan Province, Changsha 410078, Hunan, China

Correspondence to

Dr. Yue-Qiu Tan and Dr. Juan Du, Institute of Reproductive and

Stem Cell Engineering, School of Basic Medical Science, Central

South University, Changsha

410078, China;

tanyueqiu@csu.edu.cn,

tandujuan@csu.edu.cn

W-BH and CT contributed equally.

Y-QT and JD jointly supervised this work.

Received 16 December 2019

Revised 17 March 2020

Accepted 26 March 2020

D Check for updates

(c) Author(s) (or their employer(s)) 2020. Re-use permitted under CC BY-NC. No commercial re-use. See rights and permissions. Published by BMJ.

To cite: He W-B, Tan $C$ Zhang Y-X, et al. J Med Genet Epub ahead of print: [please include Day Month Year]. doi:10.1136/

jmedgenet-2019-106789

\section{ABSTRACT}

Background The genetic causes of the majority of cases of female infertility caused by premature ovarian insufficiency (POI) are unknown.

Objective To identify the genetic causes of POI in 110 patients.

Methods Whole-exome sequencing was performed on 110 patients with POI, and putative diseasecausative variants were validated by Sanger sequencing. Bioinformatic and in vitro functional analyses were performed for functional characterisation of the identified candidate disease-causative variants.

Results We identified two homozygous variants (NM_001040274: c.150_151del (p.Ser52Profs*7), c.999A > G (p.lle333Met)) in SYCP2L in two patients, which had co-segregated with $\mathrm{POI}$ in these families. Bioinformatic analysis predicted that the two variants are deleterious, and in vitro functional analysis showed that mutant SYCP2L proteins exhibited mislocalisation and loss of function.

Conclusions $S Y C P 2 L$ is a novel gene found to be responsible for human POI. Our findings provide a potential molecular marker for POI and improve the understanding of the genetic basis of female infertility.

\section{INTRODUCTION}

Premature ovarian insufficiency (POI) is characterised by the depletion or loss of normal ovarian function in women under 40 years of age; it is a frequent cause of female infertility. ${ }^{1}$ POI typically manifests with at least a 4-month history of oligomenorrhoea/amenorrhoea and elevated plasma levels of follicle-stimulating hormone ( $\mathrm{FSH}$; $>25 \mathrm{IU} / \mathrm{L})^{2}{ }^{2}$ The aetiologies of POI can be classified as genetic, autoimmune or other factors (eg, environmental factors and iatrogenic factors) ${ }^{3}$; however, most POI cases are idiopathic, with only $20 \%-25 \%$ being associated with a definitive genetic cause. ${ }^{4}$

During meiosis, diploid germ cells undergo two reducing divisions to generate haploid oocytes, which then progress through the initial stages of the first meiotic prophase and arrest at the diplotene stage prior to ovulation. ${ }^{5}$ Several studies have demonstrated that variants in meiosis-associated genes such as DMC1, PSMC3IP, TEX11 and $M E I O B$ can result in oocyte loss and cause infertility in humans. ${ }^{6-9}$ SYCP2L (SYCP2-like) is an SYCP2 homologue that is involved in synaptonemal complex (SC) assembly during the prophase of meiosis. Although Sycp2l knockout (KO) mice exhibit accelerated reproductive ageing, it is unknown whether variants in $S Y C P 2 L$ affect female fertility. ${ }^{10}$

Herein, we performed whole-exome sequencing (WES) using samples from 110 patients with POI and identified two SYCP2L variants in two individuals from two unrelated consanguineous families. Bioinformatics and in vitro functional analyses were performed to evaluate the effect of the variants on SYCP2L proteins. This is the first report of $S Y C P 2 L$ variants associated with human POI.

\section{MATERIALS AND METHODS \\ Patients}

A total of 110 Chinese women, including 5 patients from consanguineous families, were enrolled in this study after providing informed written consent. All patients were diagnosed with POI according to previously described criteria: oligomenorrhoea/amenorrhoea for at least 4 months, and an elevated FSH level $>25 \mathrm{IU} / \mathrm{L}$ on two occasions $>4$ weeks apart. ${ }^{2}$

All patients have a normal 46,XX karyotype and FMR1 CGG repeats in the normal polymorphic range. They did not exhibit any associated endocrinopathies or autoimmune disorders.

\section{WES and variant filtering}

Genomic DNA from peripheral blood samples was extracted using a QIAamp DNA blood midi kit (Qiagen, Hilden, Germany) according to the manufacturer's protocol. The core members of the consanguineous families, including parents and sisters, and the probands of non-consanguineous families were subjected to WES by the Beijing Genome Institute, as described previously. ${ }^{11}$ Identification of candidate pathogenic variants was performed as described previously, and was detailed in online supplementary methods. ${ }^{6}{ }^{11}$ The pathogenicity of the variations was evaluated according to the American College of Medical Genetics and Genomics standards and guidelines for the interpretation of variations. ${ }^{12}$

The suspected variants in $S Y C P 2 L$ were confirmed by Sanger sequencing using the primer pairs shown in online supplementary table 1 . Bioinformatics analysis is detailed in online supplementary methods.

\section{Expression of human SYCP2L}

Total RNA was extracted from the peritumoral tissues of several patients with cancer after signing 
informed written consent, including heart, liver, kidney, lung, testis and ovary. Metaphase I (MI), metaphase II (MII) and germinal vesicle $(\mathrm{GV})$ oocytes were collected from couples who agreed to donate them for research after informed consent.

Expression of the human candidate gene (SYCP2L) was evaluated by reverse transcription PCR (RT-PCR)with a specific primer (online supplementary table 1 ).

\section{Western blot analysis and immunofluorescence analysis}

Plasmid construction are described in online supplementary methods. Chinese hamster ovary $(\mathrm{CHO})$ cells were transiently transfected with expression vectors containing wild-type or mutated SYCP2L using Lipofectamine 3000 (Thermo Fisher Scientific, Carlsbad, California, USA) according to the manufacturer's instructions. Cells transfected with an empty vector (without SYCP2L) served as the negative control.

Cells were harvested 48 hours after transfection and western blot analysis is detailed in online supplementary methods, and anti-V5 antibody (Cell Signaling Technology, Danvers, Massachusetts, USA) was used as primary antibody.

Immunofluorescence analysis was performed on the transfected CHO cells grown on coverslips with anti-V5 antibody (1:800 dilution) and anticentromere (1:500, Immunovision, Springdale, Arkansas, USA) primary antibodies. Fluorescent images were captured by confocal microscopy (Olympus FV1000, Tokyo, Japan).

\section{RESULTS}

\section{Identification of SYCP $2 L$ variants}

The raw data collected by WES had a mean depth of 110.24-fold to 151.46-fold for the target regions, indicating the high sequencing quality. The WES dates of family P0001 and family P0005 are shown in online supplementary table 2. In two consanguineous families (family P0001 and family P0005), the proband, parents and sisters were subjected to WES. According to the filtering criteria, a homozygous frameshift variant of SYCP2L (NM 001040274: c.150_151del, p.Ser52Profs*7) was identified in the proband of family P0001, and a homozygous missense variant (NM_001040274: c.999A>G, p.Ile333Met) was found to be present in the proband of family P0005 (online supplementary table 3 ). Their parents and mothered sisters were carriers (figure 1A). In addition, there is no rare variant of $S Y C P 2 L$ identified in the other patients.

\section{Expression of human SYCP2L}

RT-PCR showed that human SYCP2L was specifically expressed in the testis, ovary and oocytes (GV, MI and MII oocytes; figure 2A).

\section{Phenotype of patients with the SYCP2L variant}

The proband (IV-2) from family P0001, a woman aged 31 years, was admitted to the clinic because of primary infertility (figure 1A). Her parents were first cousins. She was diagnosed with primary infertility after $>3$ years of marriage. She exhibited normal growth and development but was diagnosed with POI at 30 years of age with clinical symptoms of secondary amenorrhoea. Transvaginal ultrasound examination showed that she had a normal uterus and small bilateral ovaries, with no detectable antral follicle. Her hormonal profile showed high FSH and luteinising hormone levels as well as low serum anti-Müllerian hormone (AMH) and E2 levels (online supplementary table 4).

The proband (IV-2) of family P0005 (figure 1A), a woman aged 31 years, was the daughter of a consanguineous marriage between first cousins. She experienced highly irregular menstrual cycles (some cycles $>90$ days) at 21 and developed oligomenorrhoea at 26 years of age. At 30, she had a pregnancy that ended very early (39 days). She had elevated FSH levels and low serum AMH levels (online supplementary table 4). Transvaginal ultrasound examination revealed that the uterus and ovaries were of normal size, with three small follicles visualised in total.

Routine semen analyses of their husbands revealed normal fertility. The results of infertility-related examinations from the two patients are shown in online supplementary table 4 .

\section{Impact of SYCP2L variant}

The two variants (c.150 151del and c.999A > G) were predicted as pathogenic by three different tools, except that c.999A $>\mathrm{G}$ was predicted as a polymorphism by MutationTaster (online supplementary table 3). Multiple sequence alignment showed that amino acid p.Ile333 (c.999A) is highly conserved across species, from Xenopus tropicalis to Homo sapiens (figure 1B). A three-dimensional structure of the $\mathrm{N}$-terminal structures of SYCP2L (20-403 amino acid residues) was modelled with good confidence based on the $41.18 \%$ identical $\mathrm{N}$-terminal structures of SYCP2 (5iwz.1.A). We analysed the impact of p.Ser52Profs*7 and p.Ile333Met in SYCP2L. SWISS-MODEL revealed that the frameshift variant causes the formation of a truncated protein, with loss of $\mathrm{N}$-terminal structures, and the p.Ile333Met variant changes the shape of the protein in residues 88-95 and 205-207 (figure 1C). Meanwhile, a redundant hydrogen bond was formed between Asp334 and Ala311 when Ile333 was substituted with Met (figure 1C).

Western blot analysis showed that the mutant protein with missense variant p.Ile333Met was expressed in $\mathrm{CHO}$ cells after transfection with the expression construct (figure 2B). Although immunofluorescence analysis showed that the SYCP2L truncated protein was expressed in cells after transfection with the expression construct, western blot analysis showed that expected size of the truncated protein (p.Ser52Profs*7; 57 amino acids) was not observed (figure $2 \mathrm{~B}, \mathrm{C}$ ). It is likely that the truncated protein has reduced stability leading to its degradation and failed detection by western blot analysis. Given the variant occurs in a central exon and causes a premature stop codon, it is likely that in the in vivo setting, the transcript is degraded by nonsense-mediated decay and little of this alternative protein is encoded. As expected, immunofluorescence analysis showed that wild-type SYCP2L localised to the centromeres; however, cells transfected with the frameshift variant construct displayed cytoplasmic mislocalisation, and those transfected with the missense variant construct were not specifically localised to the centromeres, with several mutant proteins not co-localising with the centromeres (figure $2 \mathrm{C}$ ). These results demonstrate that the mutant SYCP2L proteins have altered functions. The frameshifted protein is unlikely to be expressed in patient cells due to nonsense-mediated decay, however, any residual transcript will encode a protein with impaired stability and/or function. The two variants in SYCP2L were classified as pathogenic variants.

\section{DISCUSSION}

In this study, we performed WES to reveal the genetic causes of POI in 110 patients, identifying two homozygous SYCP2L variants in two probands. Immunofluorescence revealed that the mutant SYCP2L proteins induced marked mislocalisation in vitro. To the best of our knowledge, this is the first study to report that SYCP2L is involved in POI in humans. 
A

Family P0001

Family P0005
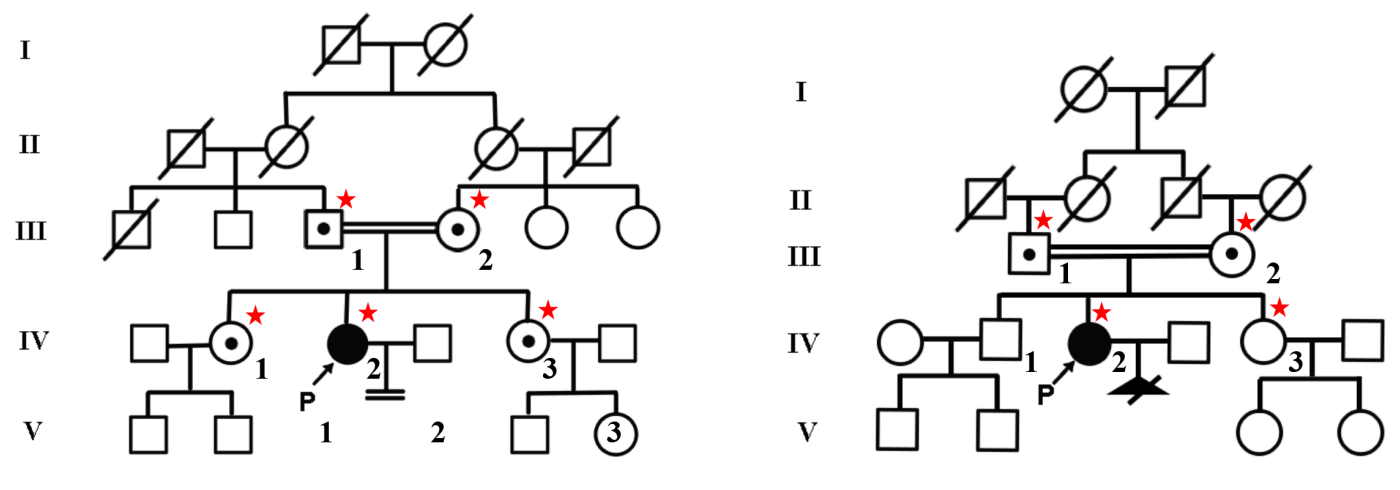

c.150_151delAG

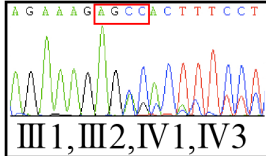

Heterozygous

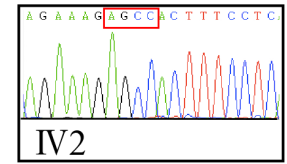

Homozygous

B

\author{
Mutated \\ H.Sapiens \\ P.Troglodytes \\ M. Mulatta \\ C. Iupus \\ B. Taurus \\ R. Norvegicus \\ G.Gallus \\ X. Tropicalis
}

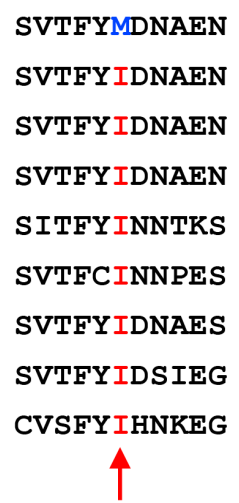

p.I333M

C

c. $999 \mathrm{~A}>\mathrm{G}$

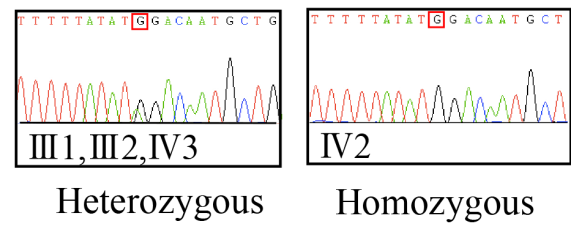

Figure 1 Pedigree and bioinformatics analysis. Affected members are indicated with filled symbols; unaffected relatives are indicated by open symbols; heterozygous carriers are indicated with a dot in the middle of the symbol. Arrows indicate the proband. Numbers are allotted to the family members whose DNA samples were used in this study; asterisks are marked on those members whose DNA was used for the whole-exome sequencing. Panel A: Sanger sequencing confirmed that in family P0001, the proband's unaffected parents (III-1 and III-2) and two mothered sisters (IV-1 and IV-3) are heterozygous carriers of the SYCP2L variant C.150_151del, whereas the proband (IV-2) is homozygous. In family P0005, the affected woman (IV-2) is homozygous for the missense SYCP2L variant C.999A>G; however, her parents (III-1 and III-2) and sister (IV-3) are heterozygous. Panel B: isoleucine at position 333 is highly conserved in different animal species from Xenopus tropicalis to Homo sapiens. Panel C: the molecular structures of the wild-type and mutant SYCP2L proteins in the N-terminal region were modelled using SWISS-MODEL software, which revealed that the frameshift variant (c.150_151del) causes the formation of a truncated protein and p.lle333Met variant (c.999A $>$ G) changes the shape of the protein at residues 88-95 (purple dashed box) and residues 205-207 (blue dashed box). Additionally, a redundant hydrogen bond was formed between Asp334 and Ala311 when lle333 was substituted by Met. 

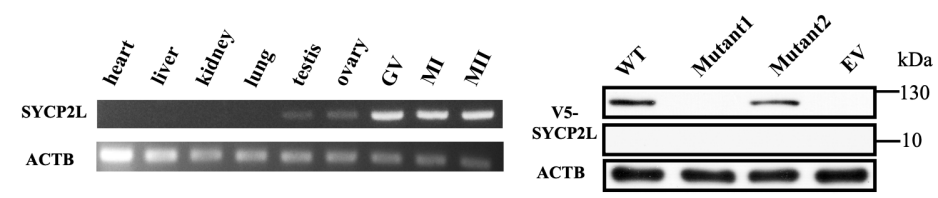

C

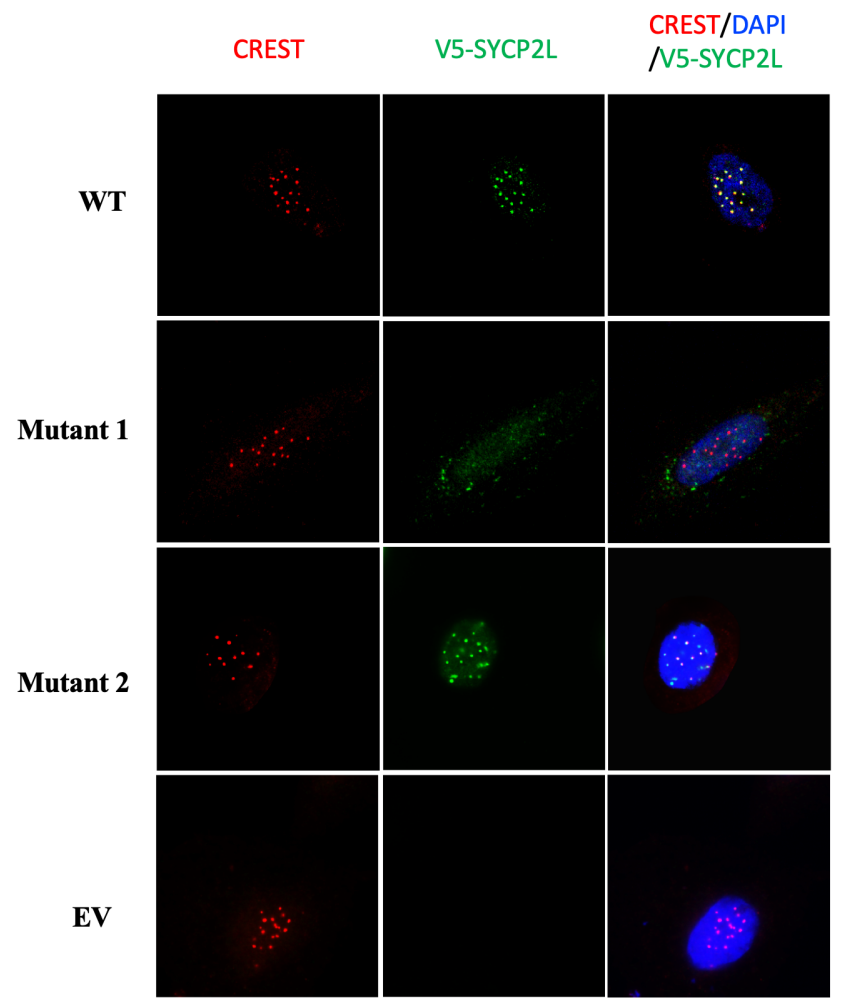

Figure 2 Human SYCP2L expression and in vitro functional analysis. Panel A: human SYCP2L is expressed in the testis, ovary and oocytes (GV, MI and $\mathrm{MII})$ of humans. Panel B: western blot analysis showing stable expression of the missense variant but no detectable truncated protein in Chinese hamster ovary $(\mathrm{CHO})$ cells after transfection. Panel C: immunofluorescence analysis demonstrating expression of both constructs. The WT SYCP2L localised to the centromeres; however, the truncated SYCP2L and missense mutant SYCP2L (p.lle333Met) displayed cytoplasmic mislocalisation and were not specifically localised to the centromeres, respectively. EV, empty vector (without SYCP2L); GV, germinal vesicle oocytes; MI, metaphase I oocytes; MII, metaphase II oocytes; mutant 1, c.150_151del of SYCP2L (p.Ser52Profs*7); mutant 2, c.999A>G (p.lle333Met) of SYCP2L; WT, wild-type SYCP2L.

The SC is required to promote synapsis and recombination during meiosis; thus, mice without SCs exhibit infertility. ${ }^{13} 14$ SYCP2L localises to the SC lateral elements in late diplotene oocytes. ${ }^{10}$ Female Sycp $2 l$-KO mice presented with subfertility. ${ }^{10}$ Recent genome-wide association studies reported that SNPs of SYCP2L (c.337-756G>A; rs2153157) may be associated with age at menarche and natural menopause in East Asian women. ${ }^{15}$ We identified two homozygous SYCP2L variants in two patients with POI presenting with secondary amenorrhoea or oligomenorrhoea and low AMH levels. The clinical features observed in our patients were similar to the phenotypes of $S y c p 2 l$-KO mice, indirectly evidencing that SYCP2L is involved in meiotic synapsis, recombination and regulating ovarian function in humans.

SYCP2L is a sequence homologue of the SC protein SYCP2, with their $\mathrm{N}$-terminals (amino acids 27-406) exhibiting 41\% identity and showing expression at the nuclear SC in oocytes beginning in the late diplotene stage, as well as localisation to centromeres in late diplotene stage oocytes. ${ }^{10}$ In vitro, ectopically expressed human wild-type SYCP2L localised to centromeres in transfected somatic cells, whereas the human truncated SYCP2L mutants were not specifically localised to the centromeres but distributed diffusely throughout the nucleus. ${ }^{10}$ In our study, the SYCP2L frameshift variant (c.150_151delAG) resulted in low expression of truncated protein in cells after transfection, likely due to its reduced stability. In vivo, it is likely the frameshift variant results in a remarkably reduced level of SYCP2L transcript due to nonsense-mediated decay, and a subsequent deficiency of SYCP2L protein in patient cells. Our experiments demonstrate that if residual transcript encodes truncated protein, it is likely unstable and/or has impaired function. The region in which the missense variant of family P0005 (c.999A > G, p.Ile333Met) is located and the amino acid itself are highly conserved, indicating that it might have an essential function. Furthermore, in vitro analysis showed that wild-type SYCP2L specifically localised to the centromeres, whereas the mutant SYCP2L proteins did not, suggesting that mutant SYCP2L proteins cause mislocalisation and ultimately altered function. Therefore, the two SYCP2L variants identified are deleterious and disease-associated variants.

In $S y c p 2 l$-KO mice, the mutant female mice completely lost Sycp 21 function and its primordial follicles were progressively lost with increasing age, leading to subfertility. ${ }^{10}$ In our study, the patient from family P0001 developed secondary amenorrhoea 
at age 30 years, whereas the patient from family P0005 experienced oligomenorrhoea and had a pregnancy at the age of 30 years. The severity of clinical symptoms of the two patients can be explained by the fact that the frameshift variant in family P0001 was a complete loss-of-function variant, but the missense variant caused partial loss of SYCP2L function, as confirmed by in vitro analysis.

We showed that human SYCP2L was expressed in both the testis and ovaries, suggesting that SYCP2L deficiency might affect both male and female fertility. Since no males in our study were homozygous for the SYCP $2 L$ variant, the effect of SYCP $2 L$ variants on human male infertility remains unclear.

In conclusion, we identified two homozygous POI-causing $S Y C P 2 L$ variants in humans that could act as new molecular biomarkers for POI in clinical settings. Our findings improve the understanding of the genetic basis of female infertility.

Acknowledgements The authors would like to thank all individuals who participated in this study.

Contributors $\mathrm{Y}-\mathrm{QT}$, JD designed the research; $\mathrm{W}$ - $\mathrm{BH}$ analysed the data and wrote the paper; $\mathrm{W}-\mathrm{BH}, \mathrm{Y}-\mathrm{XZ}$, L-LM and CT performed the research; FG, G-XL and GL performed the clinical work.

Funding This study was supported by grants from the National Natural Science Foundation of China (81771645 and 81971447), Hunan Provincial Natural Science Foundation of China (2019JJ51006), the Key Grant of Prevention and Treatment of Birth Defect from Hunan Province (2019SK1012), and the Research Grant of CITICXiangya (YNXM-201912)

Competing interests None declared.

Patient consent for publication Not required.

Ethics approval This study was approved by the Ethics Committee of the Reproductive and Genetic Hospital of CITIC-Xiangya of Central South University.

Provenance and peer review Not commissioned; externally peer reviewed.

Open access This is an open access article distributed in accordance with the Creative Commons Attribution Non Commercial (CC BY-NC 4.0) license, which permits others to distribute, remix, adapt, build upon this work non-commercially, and license their derivative works on different terms, provided the original work is properly cited, appropriate credit is given, any changes made indicated, and the use is non-commercial. See: http://creativecommons.org/licenses/by-nc/4.0/.

ORCID iD

Yue-Qiu Tan http://orcid.org/0000-0002-8359-4654

\section{REFERENCES}

1 Nelson LM. Clinical practice. primary ovarian insufficiency. N Engl J Med 2009;360:606-14
2. Webber L, Davies M, Anderson R, Bartlett J, Braat D, Cartwright B, Cifkova R, de Muinck Keizer-Schrama S, Hogervorst E, Janse F, Liao L, Vlaisavljevic V, Zillikens C, Vermeulen N, European Society for Human Reproduction and Embryology (ESHRE) Guideline Group on POI. ESHRE guideline: management of women with premature ovarian insufficiency. Hum Reprod 2016;31:926-37.

3 Goswami D, Conway GS. Premature ovarian failure. Hum Reprod Update 2005;11:391-410.

4 Qin Y, Jiao X, Simpson JL, Chen Z-J. Genetics of primary ovarian insufficiency: new developments and opportunities. Hum Reprod Update 2015;21:787-808.

5 Dutta S, Burks DM, Pepling ME. Arrest at the diplotene stage of meiotic prophase I is delayed by progesterone but is not required for primordial follicle formation in mice. Reprod Biol Endocrinol 2016;14.

6 He W-B, Tu C-F, Liu Q, Meng L-L, Yuan S-M, Luo A-X, He F-S, Shen J, Li W, Du J, Zhong C-G, Lu G-X, Lin G, Fan L-Q, Tan Y-Q, FS H. DMC1 mutation that causes human non-obstructive azoospermia and premature ovarian insufficiency identified by wholeexome sequencing. J Med Genet 2018;55:198-204.

7 Al-Agha AE, Ahmed IA, Nuebel E, Moriwaki M, Moore B, Peacock KA, Mosbruger T, Neklason DW, Jorde LB, Yandell M, Welt CK. Primary ovarian insufficiency and azoospermia in carriers of a homozygous PSMC3IP stop gain mutation. J Clin Endocrinol Metab 2018;103:555-63.

8 Caburet S, Todeschini A-L, Petrillo C, Martini E, Farran ND, Legois B, Livera G, Younis JS, Shalev S, Veitia RA. A truncating MEIOB mutation responsible for familial primary ovarian insufficiency abolishes its interaction with its partner SPATA22 and their recruitment to DNA double-strand breaks. EBioMedicine 2019;42:524-31.

9 Yatsenko AN, Georgiadis AP, Röpke A, Berman AJ, Jaffe T, Olszewska M, Westernströer B, Sanfilippo J, Kurpisz M, Rajkovic A, Yatsenko SA, Kliesch S, Schlatt S, Tüttelmann F. $X$-Linked TEX11 mutations, meiotic arrest, and azoospermia in infertile men. $N$ Eng/ J Med 2015;372:2097-107.

10 Zhou J, Stein P, Leu NA, Chmátal L, Xue J, Ma J, Huang X, Lampson MA, Schultz RM, Wang PJ. Accelerated reproductive aging in females lacking a novel centromere protein SYCP2L. Hum Mol Genet 2015;24:6505-14.

11 He W-B, Du J, Yang X-W, Li W, Tang W-L, Dai C, Chen Y-Z, Zhang Y-X, Lu G-X, Lin G, Gong $F$, Tan $Y$-Q. Novel inactivating mutations in the FSH receptor cause premature ovarian insufficiency with resistant ovary syndrome. Reprod Biomed Online 2019;38:397-406.

12 Richards S, Aziz N, Bale S, Bick D, Das S, Gastier-Foster J, Grody WW, Hegde M, Lyon E, Spector E, Voelkerding K, Rehm HL, ACMG Laboratory Quality Assurance Committee. Standards and guidelines for the interpretation of sequence variants: a joint consensus recommendation of the American College of medical genetics and genomics and the association for molecular pathology. Genet Med 2015;17:405-23.

13 Kouznetsova A, Benavente R, Pastink A, Höög C. Meiosis in mice without a synaptonemal complex. PLoS One 2011;6:e28255.

14 Cahoon CK, Helm JM, Libuda DE. Synaptonemal Complex Central Region Proteins Promote Localization of Pro-crossover Factors to Recombination Events During Caenorhabditis elegans Meiosis. Genetics 2019;213:395-409.

15 Shi J, Zhang B, Choi J-Y, Gao Y-T, Li H, Lu W, Long J, Kang D, Xiang Y-B, Wen W, Park SK, Ye X, Noh D-Y, Zheng Y, Wang Y, Chung S, Lin X, Cai Q, Shu X-O. Age at menarche and age at natural menopause in East Asian women: a genome-wide association study. Age 2016;38:513-23. 